\title{
Analyse De La Contribution Des Collectivités Territoriales Au Développement Local En Côte D'ivoire : Cas Du Département D'agboville
}

\author{
N'tchuvi Tanoh Yves-Cyrille, (Doctorant) \\ Professeur Aloko-N'guessan Jérôme \\ Géographie Humaine et Economique / Institut de Géographie Tropicale \\ (IGT), UFR des Sciences de l'Homme et de la Société, \\ Université Félix Houphouët-Boigny d'Abidjan, Côte d'Ivoire
}

Doi:10.19044/esj.2018.v14n32p286 URL:http://dx.doi.org/10.19044/esj.2018.v14n32p286

\begin{abstract}
This study focuses on the contribution of local authorities to the development of the Agboville department. It allows the two (02) levels of communities involved in the planning and development of that department. These are the former general counsel of Agboville and the regional council of Agneby-Tiassa. The first was involved in the planning and development department said 2002 to 2012. The second takes over from 2013. The main objective of this study is to highlight the ability of these local entities to provide basic services to the population for their well-being.

The methodological approach was to assess the triennial programs and administrative accounts of local authorities and conducting field surveys, to conduct interviews with local authorities and direct observation of the actions carried. The results show that investments by local authorities are poor. This deficiency is linked to the lack of financial resources, which therefore does not allow them to ensure adequate local development.
\end{abstract}

Keywords: contribution, local authorities, local development, Agboville, financial resources.

\section{Résumé}

La présente étude porte sur la contribution des collectivités territoriales au développement du département d'Agboville. Elle s'intéresse aux deux (02) échelons de collectivités qui sont l'ex conseil général d'Agboville et l'actuel conseil régional de l'Agnéby-Tiassa. Le premier a participé à l'aménagement et au développement dudit département de 2002 à 2012. Le second assure la relève depuis 2013. L'objectif principal de cette étude est de mettre en 
évidence la capacité de ces entités locales à fournir les services de base aux populations pour leur bien-être.

La démarche méthodologique a consisté à exploiter les programmes triennaux et comptes administratifs des collectivités territoriales, à réaliser des entretiens avec les autorités locales et une observation directe des actions réalisées. Les résultats montrent que les investissements réalisés par les collectivités territoriales en vue du développement du département d'Agboville sont insuffisants : l'ex conseil général a investi 1,885 milliards dans sept grands domaines et le conseil régional y a investi 2,677 milliards. Ces investissements ne peuvent couvrir les besoins des populations locales. L'insuffisance de ces investissements est liée à la faiblesse de leurs ressources financières qui de ce fait, ne leur permet pas d'assurer un développement local adéquat.

Mots clés : contribution, collectivités territoriales, développement local, Agboville, ressources financières

\section{INTRODUCTION}

La question de la gouvernance par les collectivités territoriales en Afrique est apparue nécessaire, selon Yatta (2009), après l'échec de la longue période de gestion centralisée des affaires publiques des années postindépendances. Avec son lot de réalisation de biens et de services sans réelle contribution des populations, cette gestion centralisée des politiques publiques a engendré des inégalités importantes de revenus, d'accès au logement, aux services essentiels comme l'eau, l'électricité, la santé, l'éducation, etc. Face à la persistance de cette inégalité, les préoccupations locales sont apparues de plus en plus évidentes dans ces différents pays. Le concept du local a pris de plus en plus de l'importance dans les stratégies de développement avec l'apparition d'une autre nouveauté, le concept de développement participatif dans les théories et pratiques du développement (Yatta, 2015).

Selon Hounsounon (2016), on a ainsi aboutit à la décentralisation qui a pour but de garantir l'existence de forts systèmes de protection sociale, tout en assurant l'accès de tous à des services sociaux de qualité, et notamment une amélioration du bien-être de la population.

Démarrée effectivement en Côte d'Ivoire en 1980 avec la création de 37 communes de pleins exercices, la décentralisation a porté le nombre de commune à 197 en 1997 (Loba, 2008). Puis, l'avènement d'un autre échelon de collectivités territoriales en 2002 marqua un tournant décisif à ce long processus. A partir de 2012, l'on enregistre la présence de trois échelons de collectivités territoriales dans le processus de développement local en Côte d'Ivoire : la commune, la région et le district. Les conseils généraux ont été 
supprimés au profit des conseils régionaux, organes permanents des collectivités régionales.

Cette étude met en évidence la capacité de ces entités locales à fournir les services de base aux populations pour leur bien-être. Elle porte sur le département d'Agboville dont la réalisation des actions de développement est actuellement dévolue au conseil régional de l'Agnéby-Tiassa. Toutefois, elle prend également en compte les réalisations du conseil général d'Agboville de 2002 à 2012. Ce travail permet de passer en revue les actions de développement réalisées par ceux-ci.

\section{METHODES}

Cette recherche permet d'identifier les domaines dans lesquels les réalisations ont été entreprises par les collectivités territoriales et d'analyser les budgets globaux et les montants investis dans chaque secteur pour montrer la capacité d'investissements des collectivités territoriales.

Pour parvenir à ces objectifs, la méthodologie s'est basée sur l'exploitation des programmes triennaux où sont consignées les actions de développements à réaliser et les comptes administratifs qui contiennent les actions de développement initiées, les coûts des réalisations. Cette phase a été complétée par un entretien réalisé avec le Directeur de Cabinet du Président du conseil régional. L'Adjoint du Directeur Général d'Administration, les Directeurs des services financiers et comptables, des services techniques et des moyens ont également été interviewés. Cette investigation s'est achevée par la phase de visite de terrain pour nous rendre compte de l'effectivité des réalisations et leur état de fonctionnement.

Les investissements de ces collectivités dans le département d'Agboville se rapportent aux bornes chronologiques suivantes :

- 2006-2012 pour l'ex conseil général d'Agboville (en raison de l'indisponibilité des comptes administratifs de 2002-2005) ;

- 2013-2016 pour le conseil régional.

Les réalisations ont concerné sept (07) grands domaines que sont : la santé, l'éducation, l'hydraulique, la voirie, l'électrification, le commerce et le domaine de l'administration composé des secteurs de la sécurité, de la culture.

Notons que l'insuffisance de statistiques précises et ponctuelles des comptes administratifs des conseils général et régional et l'indisponibilité des comptes administratifs de 2002 à 2005 et 2010 du conseil général ont constitué les obstacles majeurs au bon déroulement de cette recherche. 


\section{RESULTATS}

1. Les fonds investis par chaque collectivité territoriale et les domaines d'actions de développement

Les deux collectivités territoriales ont engagé 4,562 milliards au titre des investissements pour la réalisation des actions de développement dans le département d'Agboville de 2006 à 2016 (voir figure 1).

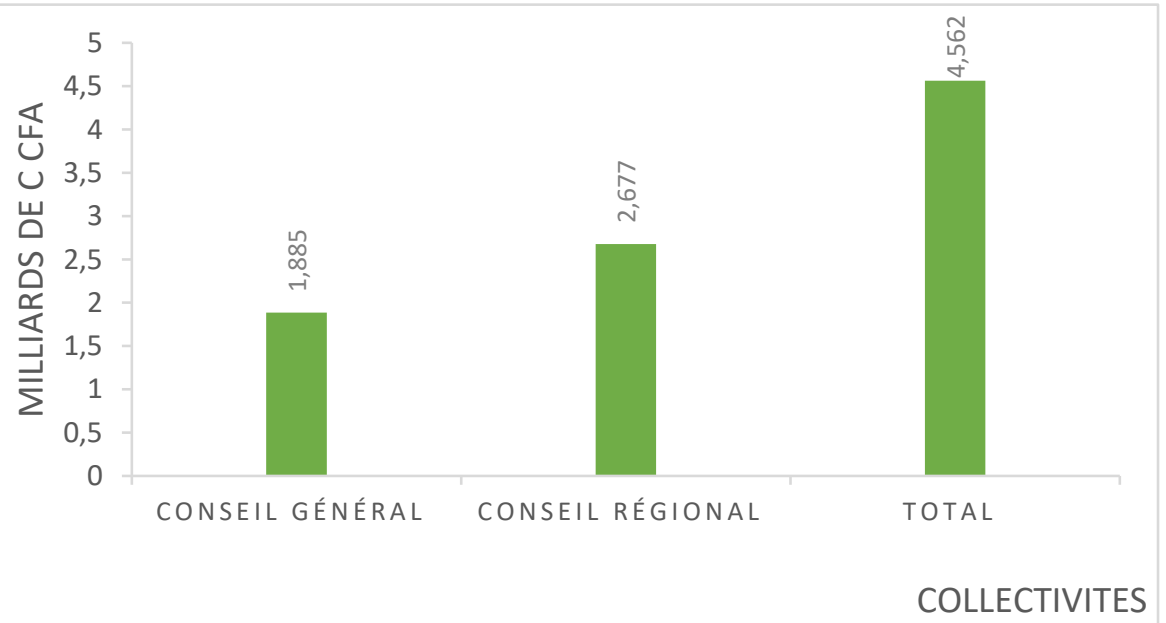

Figure 1. Fonds investis par chaque collectivité territoriale (sources de données : comptes administratifs)

Les dépenses effectuées par l'ex conseil général du département d'Agboville au titre des investissements sont estimées à 1,885 milliards F CFA.

Quant aux dépenses du conseil régional, elles sont estimées à 2,677 milliards F CFA durant la période 2013-2016.

Les fonds investis dans le département d'Agboville ont concerné divers domaines de développement. La répartition des réalisations et des actions de développement par domaine est présentée dans la partie ci-après.

\section{Le domaine de la santé}

Au total 786,849 millions de F CFA qui ont été engagés de 2006 à 2016 par les deux collectivités (voir figure 2). Le conseil général a investi 447, 949 millions représentant $57 \%$ des fonds. Mais de façon évolutive, les budgets alloués dans ce secteur ont connu une baisse : 126,2 millions de F CFA en 2006 à 97,4 millions de $\mathrm{F}$ en 2012. Le conseil régional y a investi 338,9 millions de F CFA, soit $43 \%$.

Les réalisations ont concerné des constructions de centres de santé à Grand-Yapo, Allany et de logements du personnel médical, la réhabilitation des centres de santé d'Attobrou, de Guessiguié, Yapo-Gare. Il y a eu également l'acquisition en matériels biomédicaux et d'ambulances pour les centres d'Attobrou et d'Aboudé-Kouassikro. 


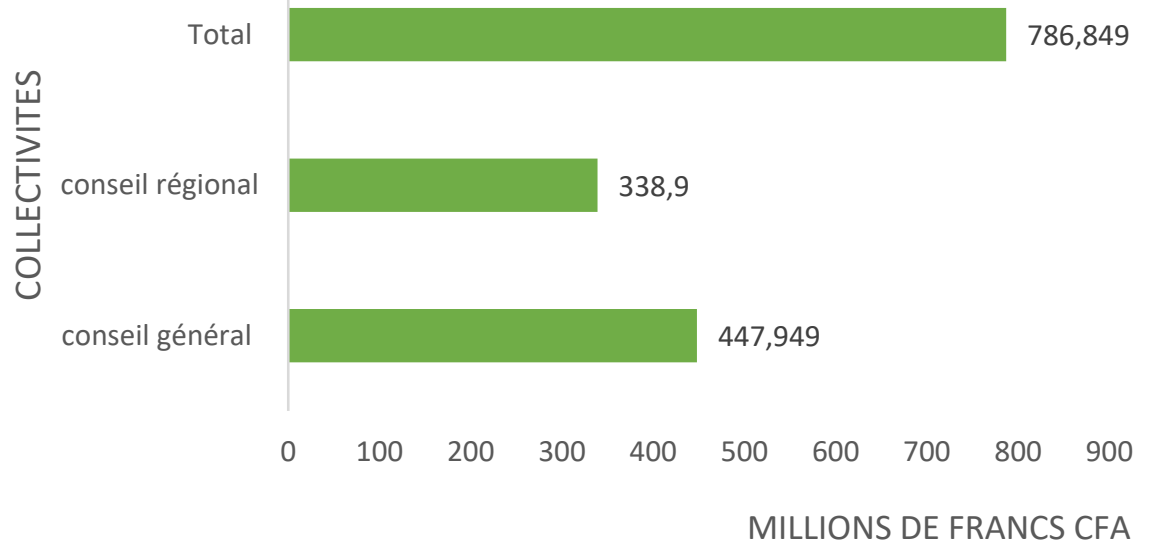

Figure 2. Fonds investis par collectivité dans la santé (sources de données : comptes administratifs)

\section{Le domaine de l'éducation}

Les fonds investis de 2006 à 2016 dans ce domaine par les deux collectivités sont estimés à 1,418 milliards de F CFA de 2006 à 2016 (voir figure 3).

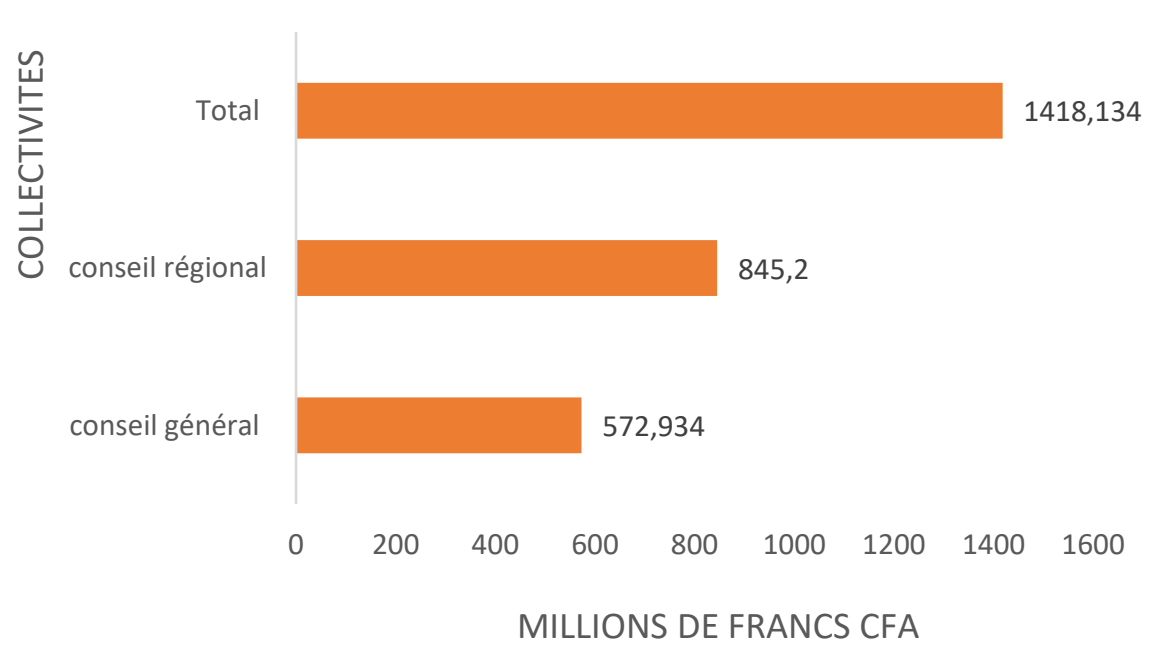

Figure 3. Fonds investis par les collectivités dans l'éducation (sources de données : comptes administratifs des conseils général et régional)

Avec 572,934 millions $\mathrm{F}$ CFA, les fonds du conseil général représentent $40 \%$ du montant global. Cette somme représente 30,40\% des fonds investis dans tous les domaines par le conseil général faisant de l'éducation, le secteur le plus pourvu financièrement par le conseil général. Les budgets alloués à ce secteur ont connu dans l'ensemble une régression : de 159, 
9 millions de F CFA en 2006 à 36,9 millions de F CFA en 2012. Dans ce secteur, le conseil régional s'est plus illustré. Il y a injecté 32,8\% des budgets d'investissements de toute la région de l'Agnéby-Tiassa, soit 1,7 milliards $F$ CFA. Dans ce secteur, le département d'Agboville détient la palme d'or avec $50 \%$ des investissements effectués, soit 845,2 millions de F CFA.

Les réalisations dans ce secteur sont relatives à la construction de salles de classes et bureaux, de blocs de latrines et la réhabilitation de logements des enseignants du primaire. Au secondaire, il s'est agi de la construction du Collège Moderne d'Attobrou, de bâtiments de salles de classes, de l'équipement des Lycées Modernes d'Agboville et d'Azaguié en tables bancs etc.

\section{Le domaine de la voirie}

Les autorités locales ont mis un point d'honneur dans le cadre des investissements de projets de développement dans ce secteur. Les fonds investis dans le secteur de la voirie sont estimé à 1,045 milliards de F CFA de 2006 à 2016 (figure 4).

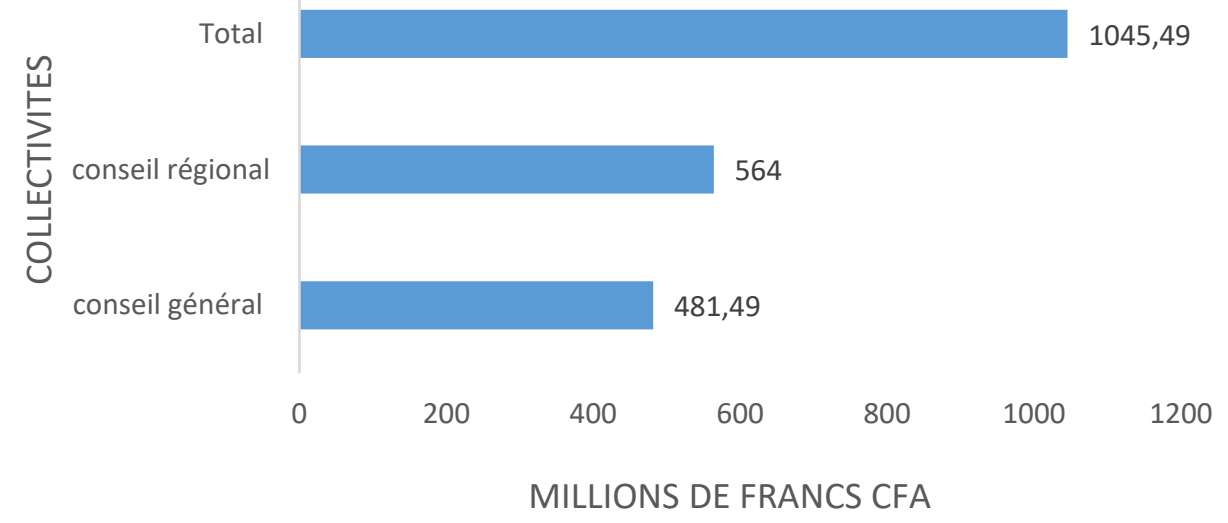

Figure 4. Fonds investis par collectivité dans l'éducation (sources de données : comptes administratifs des conseils général et régional)

Le conseil général y a investis 481,49 millions de F CFA, soit $46 \%$ du budget global d'investissement routier. Ce montant représente $25,54 \%$ de tout le budget d'investissement de 2006 à 2012. Les budgets ont connu dans l'ensemble une légère hausse passant de 80,1 millions de $\mathrm{F}$ en 2006 à 100,2 millions de F CFA en 2012. Quant au conseil régional, c'est en somme 564 millions de F CFA qui a servi à la réalisation des projets routiers. Ce montant représente $54 \%$ des 1 milliard de $\mathrm{F}$ investis globalement.

Les réalisations dans le secteur de la voirie portent sur la construction d'ouvrages de franchissement, l'entretien des voies avec le reprofilage lourd ou léger et le traitement des points critiques et l' l'ouverture des voies. 


\section{Le secteur de l'hydraulique}

Le secteur de l'hydraulique a mobilisé 351,079 millions de F CFA. Les fonds engagés par le conseil général dans ce secteur de 2006 à 2012 sont estimés à 64,208 millions de F CFA, soit $18 \%$ du montant global des deux collectivités. Quant au conseil régional, il a alloué 286,871 millions de F CFA de 2013 à 2016 (voir figure 5).

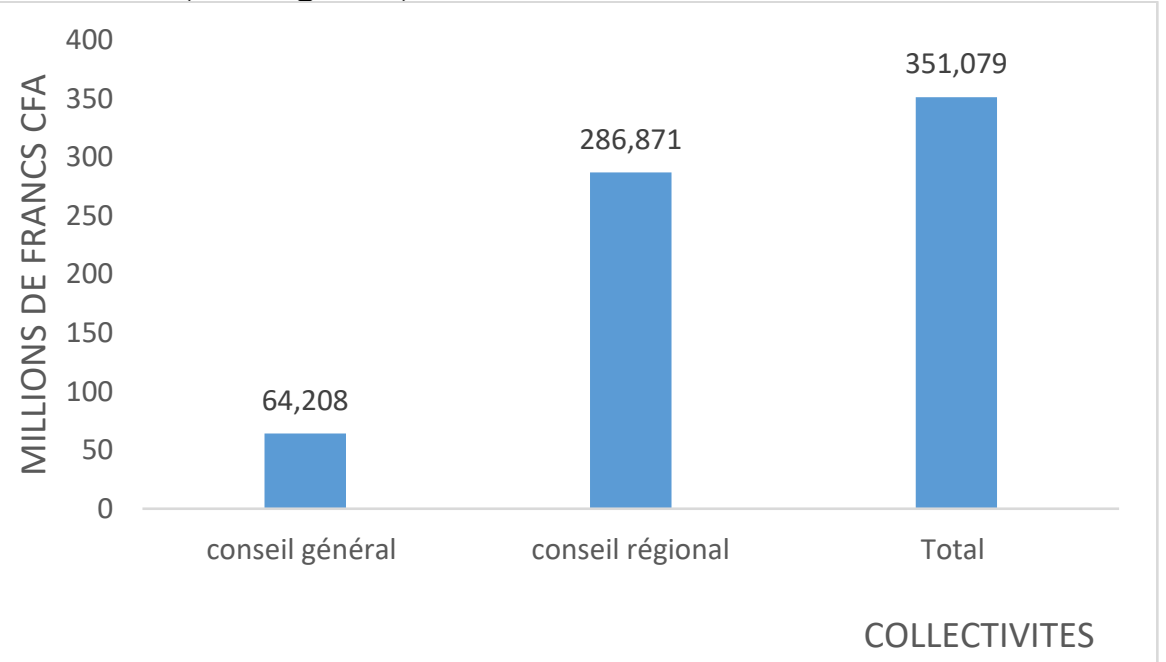

Figure 5. Fonds investis par collectivité dans l'hydraulique (sources de données : comptes administratifs des conseils général et régional)

Les travaux ont porté sur la réalisation des systèmes Hydrauliques Villageois Améliorés (HVA), de forages d'eau potables équipés de pompes à motricité humaine, à la réhabilitation des pompes d'hydrauliques villageoises, à l'extension de système hydraulique villageoise.

\section{L'électrification}

Le département a reçu $43 \%$ des 584,4 millions de F CFA affectés à ce secteur par le conseil régional, soit 251 millions de $\mathrm{F}$. Le conseil général a été passif dans l'investissement de ce secteur de 2006 à 2012.

Les travaux se résument à l'extension du réseau électrique Basse Tension (BT) dans les villages de Trénou, d'Aboudé-Kouassikro, AzguiéMakouguié, Ottopé 1, Yapokpa et le remplacement de l'Interrupteur Aérien à Commande Manuelle (AICM) du village de Grand-Yapo avec installation d'éléments connexes etc.

Le taux de couverture en électricité du département d'Agboville est passé de $22,5 \%$ en 1980 à $65 \%$ en 2000 puis à $76,30 \%$ en 2011 et $86 \%$ en 2016. Ces taux sont largement supérieurs aux taux admis au niveau national. 


\section{Le domaine du commerce}

Dans le domaine du commerce, les investissements des deux collectivités de 2006 à 2016 sont estimés à 175,86 millions de F CFA (voir figure 6).

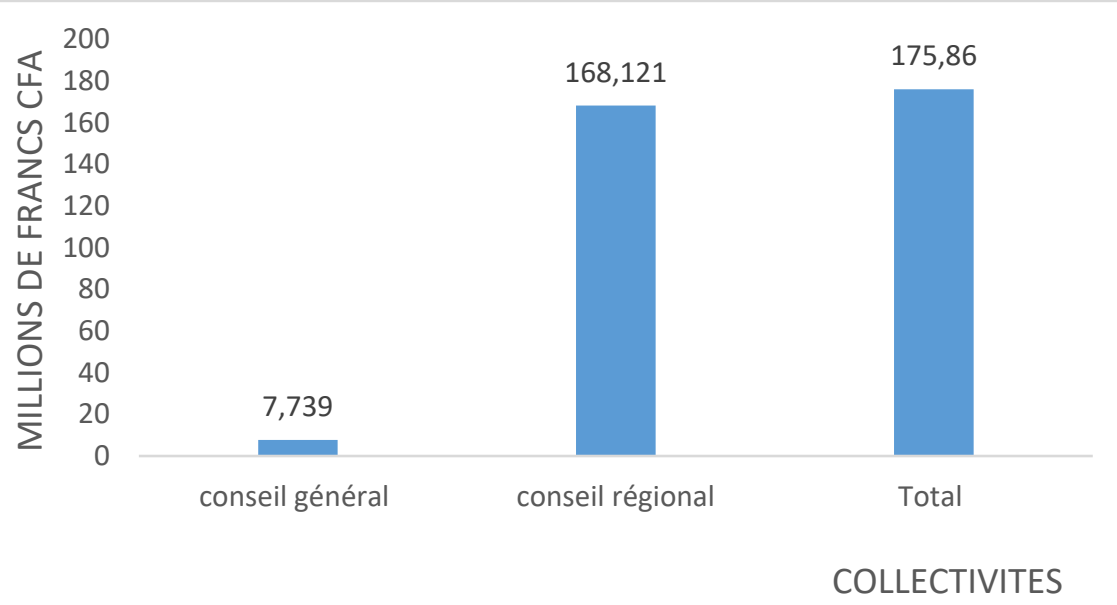

Figure 6. Fonds investis par collectivité dans le commerce (source de données : comptes administratifs des conseils général et régional)

Le conseil général s'est consacré au projet d'achèvement du marché de Grand-Morié avec un montant de 7,739 millions, soit $4 \%$ de tous les fonds investis dans ce domaine de 2006 à 2012. Ce secteur a obtenu de la part du conseil régional 168,121 millions de F CFA de 2013 à 2016, soit $96 \%$ de tous les fonds investis dans ce secteur. Les actions ont concerné la construction du marché de Grand-Morié et de 25 magasins dans ce marché.

\section{Dans l'administration}

Dans le secteur de l'administration, la réalisation des projets a principalement concerné la construction du siège du conseil général avec les équipements et le matériel y afférents. Ce fait est relatif à la recense de leur mise en place. L'acquisition de ces besoins administratifs a nécessité 148,7 millions de F CFA, soit 7,89 \% de budget global des investissements de 2006 à 2012 (voir figure 7). 


\section{Conseil général}
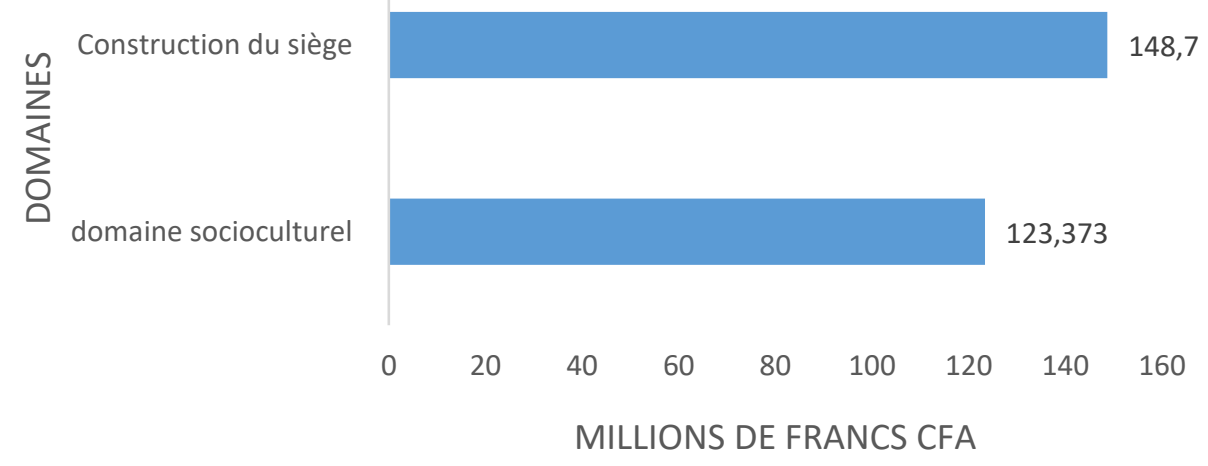

Figure 7. Fonds investis par le conseil général dans le domaine socioculturel et la construction du siège (sources de données : comptes administratifs du conseil général)

Dans le secteur socioculturel, c'est en somme un budget de 123,373 millions de F CFA qui a été alloués par le conseil général pour la réalisation des projets, soit 6,54\% des fonds investis de 2006 à 2012 (voir figure 7). Ces réalisations ont porté sur une assistance sociale effectuée à l'aide de 5 millions F CFA en 2006 et la construction et l'équipement de bâtiment abritant la radio du département d'un montant de 118,373 F engagés de 2008 à 2012. Dans ce domaine le conseil régional est resté passif dans le financement des projets concernant le département d'Agboville.

La sécurité, quant à elle, a enregistré 224,267 millions de F CFA de 2006 à 2016 (figure 8).

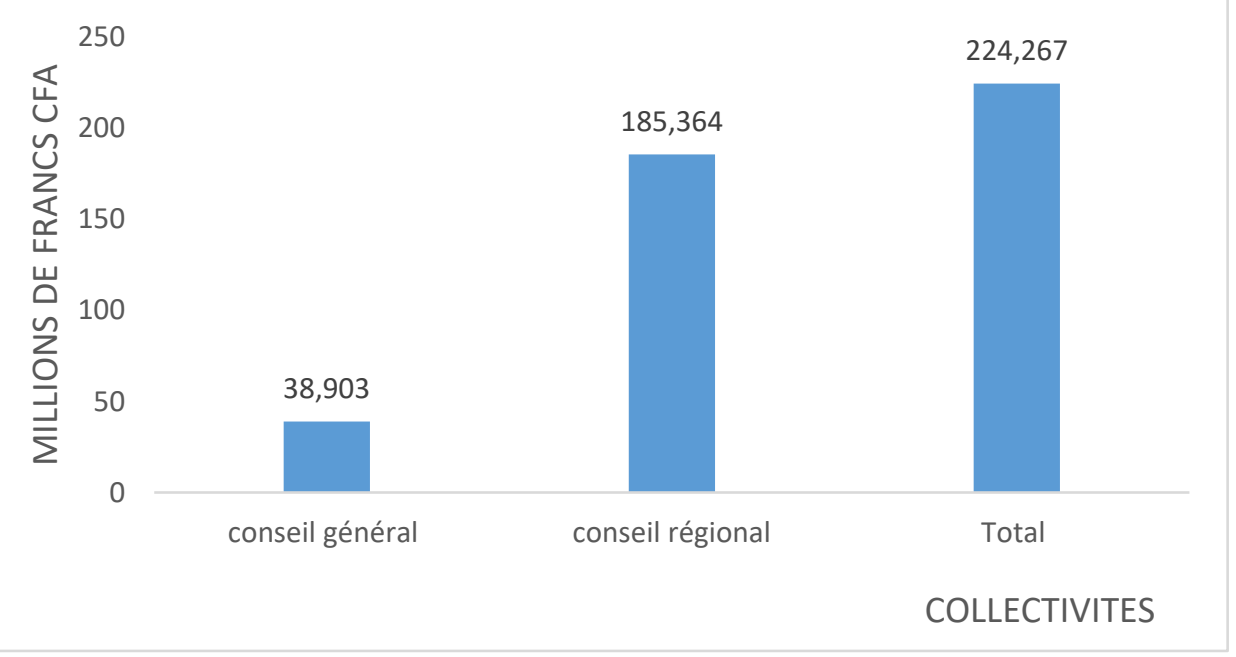

Figure 08. Fonds investis par les collectivités dans le secteur de la sécurité (source de données : comptes administratifs des conseils général et régional) 
Le conseil général y a investi 38,9 millions de $\mathrm{F}$, soit $17 \%$ des fonds investis de 2006 à 2012 contre $73 \%$ au compte du conseil régional, soit une valeur de 185,364 millions de F CFA de 2013 à 2016. Les projets ont essentiellement porté sur la construction de brigade de gendarmerie de Loviguié et de Céchi.

\section{Taux d'exécutions des recettes d'investissement recouvrées par les deux collectivités territoriales}

Tableau I. Taux d'exécution des recettes d'investissement recouvrées par le conseil général

\begin{tabular}{|c|c|c|c|c|c|c|c|}
\hline Années & $\mathbf{2 0 0 6}$ & $\mathbf{2 0 0 7}$ & $\mathbf{2 0 0 8}$ & $\mathbf{2 0 0 9}$ & $\mathbf{2 0 1 1}$ & $\mathbf{2 0 1 2}$ & Total \\
\hline $\begin{array}{c}\text { Recettes } \\
\text { d'investissement }\end{array}$ & 730,767 & 404,500 & 744,552 & 642,929 & 818,873 & 873,049 & $\mathbf{4 2 1 4 , 6 7 0}$ \\
\hline $\begin{array}{c}\text { Dépenses } \\
\text { d'investissement }\end{array}$ & 445,384 & 284,831 & 412,687 & 108,536 & 285,924 & 277,064 & $\mathbf{1 8 1 4 , 4 2 6}$ \\
\hline Taux d'exécution (\%) & 60,95 & 70,41 & 55,43 & 16,88 & 34,91 & 31,73 & $\mathbf{4 3 , 0 5}$ \\
\hline
\end{tabular}

Source : comptes administratifs

Le taux d'exécution des recettes est de 43,05\% de 2006 à 2012, soit 1,814 milliards F CFA engagés sur 4,215 milliards recouvrés (tableau I). Les taux d'exécution les plus élevés ont été enregistrés en 2006 et 2007. Ils sont respectivement de $60,95 \%$, soit 445,384 millions F CFA dépensés sur 730,767 millions F CFA recouvrés et de 70,41\%, soit 284,831 millions dépensés contre 404,500 millions recouvrés. Le taux d'exécution le plus bas est enregistré en 2009 avec $16,88 \%$, soit 108,536 millions F CFA dépensés pour 642,929 millions F CFA disponibles. En 2008, les fonds investis par le conseil général sont de 412,687 millions F CFA sur les 744,552 millions F CFA recouvrés, soit un taux d'exécution de 55,43. En 2012, le conseil général a investis 277,064 millions F CFA contre 873,049 millions F CFA disponible.

Tableau II. Taux d'exécution des recettes d'investissement recouvrées par le conseil régional

\begin{tabular}{|c|c|c|c|c|c|}
\hline Années & $\mathbf{2 0 1 3}$ & $\mathbf{2 0 1 4}$ & $\mathbf{2 0 1 5}$ & $\mathbf{2 0 1 6}$ & Total \\
\hline Recettes d'investissement recouvrées & & & & & \\
& 0,727 & 1,156 & 2,566 & 2,735 & $\mathbf{7 , 1 8 5}$ \\
\hline Dépenses d'investissement & & & & & \\
& 0,070 & 0,449 & 1,059 & 1,411 & $\mathbf{2 , 9 8 8}$ \\
\hline Taux d'exécution (\%) & 09,67 & 38,82 & 41,24 & 51,57 & $\mathbf{4 1 , 5 9}$ \\
\hline
\end{tabular}

Source : comptes administratifs

Le conseil régional a enregistré un taux d'exécution de $41,59 \%$, soit 2,988 milliards F CFA dépensés pour un total de 7,185 milliards recouvrés de 2013 à 2018. Pour avoir engagé 70 millions F CFA dans les dépenses d'investissement contre 727 millions de F CFA recouvrés, cette collectivité a enregistré un taux d'exécution de 9,67\%. En 2014, le conseil régional a dépensé 449 millions F CFA pour 1,156 milliards F CFA recouvrés, soit un taux d'exécution de $38,82 \%$. Les dépenses effectuées au tire des 
investissements en 2015 sont estimées à 1,059 milliards F CFA pour des recettes recouvrées de 2,735 milliards F CFA, soit un taux d'exécution de 41,24\% (voir tableau II). Le taux d'exécution le plus élevé est enregistré en 2016 avec une moyenne de 51,57 \%, soit 1,411 milliards F CFA dépensés sur 2,735 milliards de recettes recouvrées.

\section{Le montant d'investissement par an et par habitant}

L'effort d'investissement d'une collectivité est perçu par l'investissement annuel par habitant.

Tableau III. Montant de l'investissement par habitant et par an

\begin{tabular}{|c|c|c|c|}
\hline $\begin{array}{c}\text { Collectivités } \\
\text { territoriales }\end{array}$ & $\begin{array}{c}\text { Budget } \\
\text { d'investissement }\end{array}$ & $\begin{array}{c}\text { Effectif de } \\
\text { population }\end{array}$ & $\begin{array}{c}\text { Montant par an } \\
\text { et par habitant }\end{array}$ \\
\hline $\begin{array}{c}\text { Conseil général } \\
(2012)\end{array}$ & 873049000 & 317376 & 2750 \\
\hline $\begin{array}{c}\text { Conseil régional } \\
(2014)\end{array}$ & 1156000000 & 606852 & 1905 \\
\hline
\end{tabular}

Source : comptes administratifs conseils général et régional

L'ex conseil général a enregistré un budget d'investissement annuel de 873,049 millions F CFA pour un effectif de population départementale estimé à 317376 habitants en 2012, soit 2750 F CFA investi par an et par habitant (tableau III).

Quant au conseil régional, avec un budget d'investissement de 1,156 milliards de F CFA en 2014 pour un effectif de la population régionale estimé à 606852 habitants, il a investi $1905 \mathrm{~F} / \mathrm{an} /$ habitant.

\section{L'étroitesse des ressources propres des deux collectivités territoriales Cas du conseil régional de l'Agneby-Tiassa}

Les ressources propres du conseil régional de l'Agneby-Tiassa représentent en moyenne moins de 5\% durant l'exercice 2013-2016.

Tableau IV. Poids des recettes propres du conseil régional

\begin{tabular}{|c|c|c|c|}
\hline $\begin{array}{c}\text { Années } \\
\text { d'exercice }\end{array}$ & $\begin{array}{c}\text { Recettes totales } \\
\text { du conseil régional }\end{array}$ & $\begin{array}{c}\text { Recettes propres } \\
\text { du conseil régional }\end{array}$ & $\begin{array}{c}\text { Poids des recettes } \\
\text { propres }(\%)\end{array}$ \\
\hline $\mathbf{2 0 1 3}$ & 538215998 & 19052846 & 3,55 \\
\hline $\mathbf{2 0 1 4}$ & 741145868 & 26287492 & 3,55 \\
\hline $\mathbf{2 0 1 5}$ & 1391192961 & 17807270 & 1,28 \\
\hline $\mathbf{2 0 1 6}$ & 1192276161 & 24322435 & 2,04 \\
\hline
\end{tabular}

Source : comptes administratifs conseils général et régional

Le poids des ressources propres du conseil régional par rapport aux ressources globales de fonctionnement était de 3,4\% les deux premières années d'exercice (2013 et 2014), de 1,28\% en 2015 et de 2,04 en 2016 (tableau IV). 


\section{Cas de l'ex conseil général d'Agboville}

Mise en place effectivement en 2002, les conseils généraux ont commencé a fonctionné en 2003 à l'aide des subventions de l'Etat. Mais quelques années après, l'élaboration du budget de cette collectivité territorial a tenu compte des ressources propres locales. Le tableau $\mathrm{V}$ ci-dessous présente le poids des ressources propres durant l'exercice 2006-2012 du conseil général d'Agboville.

Tableau V. Poids des recettes propres du conseil général

\begin{tabular}{|c|c|c|c|}
\hline $\begin{array}{c}\text { Années } \\
\text { d'exercice }\end{array}$ & $\begin{array}{c}\text { Recettes totales } \\
\text { du conseil général }\end{array}$ & $\begin{array}{c}\text { Recettes propres } \\
\text { du conseil général }\end{array}$ & $\begin{array}{c}\text { Poids des recettes } \\
\text { propres (\%) }\end{array}$ \\
\hline 2006 & 423330000 & 27807000 & 06,6 \\
\hline 2007 & 307462000 & 30029000 & 09,8 \\
\hline 2008 & 275852000 & 27106000 & 09,8 \\
\hline 2009 & 281493000 & 29140000 & 10,4 \\
\hline 2011 & 229610000 & 20033000 & 08,8 \\
\hline 2012 & 246147000 & 34189000 & 13,9 \\
\hline
\end{tabular}

Source : comptes administratifs conseil général et régional

Les ressources propres du conseil général d'Agboville sont d'une extrême faiblesse bien qu'ayant connu une légère hausse. Dans l'ensemble, elles représentent en moyenne moins de $10 \%$ des ressources globales.

En 2006, les recettes propres représentaient $6 \%$ des recettes globales, $9,8 \%$ sur les deux années successives et $08,8 \%$ en 2011 (tableau V). Les années 2009 et 2012 sont celles où les recettes propres ont pesé plus de $10 \%$ des recettes globales c'est-à-dire 10,4\% en 2009 et 13,9 \% en 2012 (tableau V).

\section{Discussion}

Dans cette section, il s'agit des limites de notre étude, de l'analyse et de l'enrichissement des résultats obtenus par d'autres travaux sur la question de la capacité des collectivités territoriales à contribuer au développement local. Ces limites portent sur la fiabilité des données utilisées dans cette étude. A ce titre, les données du récent recensement sont à considérer avec beaucoup de réserve. D'un effectif de 244865 habitants en 1998, la population du département d'Agboville est estimée à 292109 habitants en 2014 (Essoh, 1998 ; INS, 2014), soit un taux d'accroissement de 1,1\%. Lors de nos enquêtes de terrain, certaines autorités administratives et leaders communautaires n'ont pas manqué de nous rappeler que nombre des populations ont refusé de se faire recenser. Ainsi, l'on serait tenté de dire que les statistiques exploitées pourraient-être sous-estimées et cacheraient des pressions démographiques plus importantes sur les services de base.

En dehors de cet aspect, les données statistiques collectées, à partir desquelles des ratios ont été obtenus, ont permis de faire une analyse objective. 
Les résultats de cette étude montrent que les actions de développement réalisées par les acteurs locaux dans divers domaines socioéconomiques sont insuffisantes.

Cette insuffisance est liée à la faiblesse des ressources financières notamment l'étroitesse des dotations budgétaires annuelles, les fonds investis par habitant et par an.

En ce qui concerne l'étroitesse des dotations budgétaires des collectivités, le conseil général a enregistré des budgets annuels de l'ordre de 1,078 milliards F CFA en moyenne. Quant au conseil régional de l'AgnebyTiassa, il enregistre un budget global annuel de 2,796 milliards en moyenne. S'agissant de l'investissement par an et par habitant, cet indicateur de mesure du niveau d'investissement d'une collectivité est relativement faible : 2750 F/an/habitant en 2012 pour l'ex conseil général d'Agboville et 1905 F/an/habitant pour le conseil régional en 2014. Ces moyennes sont de loin inférieures aux recommandations de la Direction et Contrôle des Grands Travaux (DCGTx, 1994) contenu dans le Plan d'Urbanisme Directeur d'Agboville qui stipule qu'il faudrait investir théoriquement $15.000 \mathrm{~F} / \mathrm{an} / \mathrm{habitant}$ pour suivre la croissance démographique. Même si les difficultés de l'Etat et des autorités municipales obligent à retenir $10.000 \mathrm{~F} / \mathrm{an} / \mathrm{habitant}$, les budgets d'investissement des deux (02) collectivités sont de loin inférieurs à ce barème. En principe, il aurait fallu au conseil régional un budget d'investissement annuel d'une valeur de 6,068 milliards de $\mathrm{F}$, soit le quintuple du montant disponible pour répondre aux besoins de la population régionale en termes de fourniture de service de base. Quant à l'ex conseil général, il lui aurait fallu 2,684 milliards de F, soit presque le quadruple des fonds investis pour suivre la croissance démographique du département.

La faiblesse des budgets d'investissements est liée en partie à l'étroitesse des ressources propres locales. Notre étude montre que les ressources propres locales du conseil général représentent en moyenne moins de $10 \%$ des ressources globales et celles du conseil régional, moins de $5 \%$. Au regard de ce qui précède, il était très difficile à l'ex conseil général de suivre la croissance démographique dans l'optique de réaliser des investissements conséquents en termes d'infrastructures et d'équipements socioéconomiques. Il en est de même pour le conseil régional qui assure la relève aujourd'hui. C'est une mission qui parait quasi impossible si les fonds devraient provenir du budget local, quand on sait que les besoins des populations en constante évolution se font de plus en plus sentir.

Les conséquences de la faiblesse des ressources budgétaires des collectivités territoriales se manifestent dans différents domaines par des disparités et des déséquilibres dans l'accès aux équipements de bases.

Dans le domaine de la santé, l'insuffisance des structures sanitaires et du personnel médical est remarquable. Tandis que l'OMS recommande 1 
Hôpital de référence en l'occurrence un Hôpital Général ou un CHR (Centre Hospitalier Régional) pour 150000 habitants, le département d'Agboville enregistre 1 hôpital de référence pour 311276 habitants en 2017. Au niveau des Etablissements Sanitaires de Premier Contact (ESPC), la moyenne départementale qui est de 1 ESPC pour 8894 habitants en 2017, cache des disparités au niveau des sous-préfectures. Les ratios des sous-préfectures d'Aboudé-Mandeké, d'Agboville et de Rubino sont respectivement de 1 ESPC pour 18625 habitants, 1 ESPC pour 13163 habitants et 1 ESPC pour 12419 habitants. Ces moyennes montrent combien les sous-préfectures du département d'Agboville sont sous-équipées en structures sanitaires, la norme étant de 1 ESPC pour 10000 habitants. L'insuffisance de ces structures sanitaires rend difficile l'encadrement des médecins car leur affectation en dépend. Le ratio de 1 médecin pour 11529 habitants en 2017 du département d'Agboville est en déphasage avec la recommandation de l'OMS qui est de 1 médecin pour 10000 habitants. Ce gap est plus creusé au niveau des souspréfectures. On note à cet effet, 1 médecin pour 37275 habitants dans la souspréfecture de Rubino, 1 médecin pour 27171 habitants à Azaguié, 1 médecin pour 22428 habitants à Céchi.

Dans le domaine de l'éducation, le secteur de l'enseignement reste confronté à des problèmes liés à l'encadrement des populations scolaires. Le département d'Agboville enregistrait en moyenne 45 élèves par classe en 2015 dans le primaire alors que la norme est de 33 élèves par classes. Au niveau des sous-préfectures, on note 57 élèves par classe à Céchi, 51 élèves par classe à Loviguié et Rubino. Quant au secondaire, la moyenne est de 65 élèves par classe. Il en ressort un effectif pléthorique dans les salles de classes.

Quant au domaine de l'hydraulique, le département d'Agboville est en très mauvaise posture : le déficit de production d'eau potable s'élève à 5218 $\mathrm{m}^{3}$ d'eau, soit $49 \%$ des besoins en eau potable non satisfaits. Ce déficit est aggravé au niveau de certaines zones de production. En témoignent les faibles taux de couvertures : $32 \%$ à Azaguié, $29 \%$ Aboudé-Mandeké, $28 \%$ à GrandYapo, $16 \%$ à Attobrou et $10 \%$ à Ananguié qui enregistre le taux de couverture en eau potable le plus bas.

Concernant le domaine de la voirie, le département d'Agboville compte seulement $14,25 \%$ de voies bitumées contre $85,75 \%$ en terre. Les $85,75 \%$ de voies en terre du département sont en mauvais état surtout en saison pluvieuse. Dans cet ensemble, $89 \%$ de ce linéaire connectent les campements aux villages, les villages aux chefs-lieux de sous-préfectures et dans certains cas ces derniers à la ville d'Agboville.

Ces problèmes qui résultent de l'insuffisance des fonds investis par les collectivités territoriales sont en partie liés au contexte économique et sociodémographique du département d'Agboville. 
Pour ce qui est du contexte économique, l'économie de la région est secouée par une crise des secteurs agricole, industriel et tertiaire. La crise du secteur agricole essentiellement dominé par l'économie de plantation se manifeste par la réduction des superficies cultivées et des productions. Les superficies cacaoyères ont été réduites de $65 \%$ de 2001 à 2014 passant respectivement de 57694 ha à 19805 ha. Quant aux productions, elles sont passées de $41682,86 \mathrm{~T}$ en 2001 à $14082 \mathrm{~T}$ en 2015 pour le cacao, 13 405,25 T à 1587 T pour le café la même période (Ministère de l'Agriculture, 2007 ; Conseil café-cacao, 2016). Le corolaire de la baisse des productions des cultures de rente est la faiblesse des revenus des producteurs. La crise du secteur industriel est perçue par la réduction des effectifs des employés. On est passé de 1567 salariés employés par la COTIVO en 1978 à 150 personnes en 2014 soit un licenciement de plus de $90 \%$ du personnel (Dubresson, 1981 ; Ministère du Plan et du Développement, 2015). La faiblesse des revenus du secteur agricole et le chômage enregistré dans le secteur industriel rendent difficile la participation des populations au développement de leur localité. Ce développement est encore rendu très difficile $\mathrm{du}$ fait du contexte sociodémographique du département d'Agboville.

Quant au contexte sociodémographique, le département d'Agboville connait une évolution importante de sa population caractérisée par son inégalement répartition et son extrême jeunesse. La population est passée de 148520 habitants en 1975 à 292109 habitants en 2014 (Ministère de l'Economie, des Finances et du Plan, 19812 et INS, 2014). Sa distribution par milieu d'habitat marque un déséquilibre au profit du milieu rural : $73 \%$ de la population départementale en milieu rural contre $23 \%$ en milieu urbain. Sachant que la quasi-totalité des services sont concentrés en milieu urbain, il en résulte un déséquilibre dans l'accès au service de bases. Quant à l'extrême jeunesse de la population : les moins de 20 ans représentent 51,55\% de la population totale du département en 2014. Cette extrême jeunesse de la population constitue un problème majeur pour le développement humain durable : un problème au niveau des besoins sociaux que sont l'éducation et la santé. Cela sous-entend du point de vue des politiques sociales une implication des acteurs politiques et une mobilisation des ressources en vue de répondre de façon cohérente aux besoins sociaux. Car en effet, les coûts de formation de ces flots de jeune croissent de manière exponentielle, de même que les coûts de santé et d'équipements de toute sorte.

Les résultats de cette étude montrent que les actions de développement des collectivités territoriales sont insuffisantes. Leur contribution au développement local est par conséquent faible. Ces résultats nous ont orientés vers d'autres travaux de recherche des auteurs aussi bien ivoiriens que d'autres pays. 
En Côte d'Ivoire, les études de Loba (2008), Konan (2011), Bosson (2013) ont mise en lumière l'insuffisance budgétaires des collectivités territoriales. Selon les travaux de Loba (2008), les dotations budgétaires des communes de Dabou, Bingerville et Jacqueville de 1991 à 2005, tournent en moyenne et de façon respective autour de 484 millions de F CFA, 344 millions de F CFA et 132 millions de F CFA par année d'exercice. Konan (2011) décrit une instabilité du budget global de la commune de Dimbokro qui est passé de 296 millions de F à 315 millions de F CFA de 2001 à 2006. Quant au troisième, ses résultats montrent que les budgets des conseils généraux d'Adzopé et de Bongouanou sont respectivement de 1,28 milliard et 884 millions. D'autres études réalisées montrent la généralisation de cette situation en Afrique. A partir des résultats de leurs travaux, Chambas, Brun et Graziosi (2007), font un constat global sur la faiblesse des ressources des collectivités locales d'Afrique subsaharienne. Le plus souvent, les ressources des collectivités locales s'élèvent à environ $1 \%$ du PIB. Elles s'établissent en moyenne dans les pays de l'UEMOA à 0,7\% du PIB (Burkina Faso : 0,6\% ; Côte d'Ivoire 1\% ; Sénégal, 0,8\%).

Quant à l'investissement par an et par habitant, les résultats de ZAIDCHERTOUK (2007) sont similaires aux nôtres. Selon lui, cette faiblesse d'investissements est remarquée dans les communes de la Wilaya de Tizi Ouzou en Algérie. Les communes de montagne enregistrent des taux vraiment dérisoires à l'image de la commune d'Aït Oumalou avec 90 DA/habitant [0.90 $€ /$ habitant] ou de Souk El Thenine avec $77 \mathrm{DA} /$ habitant [0.77 €/habitant] ou encore d'Assi Youcef avec 54 DA / habitant [0.54 €/habitant] en 2000. Pour permettre de mieux appréhender l'extrême faiblesse de ces fonds investis annuellement par habitant, il met en évidence celles de la commune française de Spay : $472.3 €$ /an/habitant, soit 309800 F CFA/an/habitant en 2003.

En ce qui concerne l'étroitesse des ressources propres des collectivités, les résultats des études de Ovono (2012) sur les collectivités du Gabon et de Touzi (2000) sur la Mauritanie, sont similaires aux nôtres. Pour Ovono (2012), les ressources propres des collectivités locales du Gabon sont en moyenne de $10,3 \%$ de 2001 à 2005. De façon détaillée, elles se présentent comme suit : 10 $\%$ de l'ensemble des ressources globales $2001,7 \%$ en $2002,11,5 \%$ en 2003 , $11 \%$ en 2004 et $12 \%$ en 2005 . Les ressources globales des communes de la Mauritanie s'élèvent à 1652 milliards UM (Ouguiya Mauritanien) soit environ $3 \%$ des recettes publiques ou encore $0,6 \%$ du PIB (Touzi, 2000).

S'agissant du contexte économique défavorable, les résultats des travaux de Memel (2012) sur Dabou, Bosson (2013) sur les départements d'Adzopé et de Bongouanou, Konan (2011) sur Dimbokro et de Ruf et Agkpo (2008), sur le revenu et les investissements des producteurs de café et cacao en Côte d'Ivoire, sont similaires aux nôtres. A propos de Dabou, Memel (2012), soutient que l'étroitesse des bases économiques est liée à la relative faiblesse 
des ressources financières de cette commune. Elle est confrontée à une économie agricole à faible valeur ajoutée, un secteur secondaire inexistant et un secteur tertiaire dominé par des services improductifs de l'informel. Dans le même sens, Bosson (2013) conclue que l'agriculture qui a été à l'origine des progrès du pays Akyé et du Moronou, régulièrement confrontée aux aléas climatiques (sécheresse, pluies irrégulières) et dépendante de technique encore rudimentaire : culture itinérante sur brûlis, outillage archaïque et non utilisation des engrais. Par ailleurs, du fait de la baisse des prix du binôme café-cacao, principale source de revenus des paysans, ces derniers participent difficilement au développement de leur localité. Ruf et Agkpo (2008), ont estimé à 107.000 F CFA le revenu annuel par personne et à 293 F CFA le revenu journalier par personne entre 2006 et 2007. Pour eux, tout indique que le cacao et le café n'empêchent pas le revenu par personne de tomber en dessous de 1 US \$ par personne et par jour. Il en résulte une pauvreté des exploitants.

Pour le contexte sociodémographique, les études de Ngamassu (2005), Paré-Kaboré et Gambré-Idany (2014), Loba (2009), Kouadio (2010) et Nkengfack, Domguia et Kamajou (2017). Pour Ngamassu (2005), la croissance démographique exponentielle constitue l'un des problèmes auxquels l'école est confrontée au Cameroun car elle entraine l'augmentation constante des demandes en matière d'éducation. Les salles de classe prévues pour accueillir une trentaine d'élèves il y a vingt ans en accueillent aujourd'hui deux, voire trois fois plus. Au Burkina Faso, les effectifs pléthoriques des classes sont vus comme étant un des facteurs entravant la performance du système scolaire (Paré-Kaboré et Gambré-Idany, 2014). Au niveau de la couverture sanitaire, Loba (2009) conclue que, suite à l'accroissement rapide de la charge démographique, la pression sur les équipements sanitaires a abouti à l'obtention de ratio d'encadrement médiocre. La couverture médecin par habitant est faible. L'inégale répartition de population entraine également des disparités dans l'encadrement des populations. Selon Kouadio (2010), dans le département d'Abengourou, tandis que la seule ville du même nom, avec $25 \%$ de la population, concentre $47 \%$ des structures sanitaires avec toutes les spécialités, le milieu rural enregistre $53 \%$ des centres de santé pour $75 \%$ de la population départementale. A propos de la couverture en eau potable, Nkengfack, Domguia et Kamajou (2017) montrent que c'est en Afrique subsaharienne que le taux d'accès à l'eau potable et à l'assainissement est le plus faible du monde. Seuls $46 \%$ de la population rural y ont accès.

\section{Conclusion}

Cette étude a relevé la faiblesse des actions de développement de l'ex conseil général d'Agboville et du conseil régional de l'Agnéby-Tiassa. Malgré leur contribution, le département d'Agboville est confronté à une situation de développement contrarié dont les manifestations sont perçues dans divers 
domaines notamment l'éducation et la santé. Au niveau de l'éducation, le département est confronté au caractère pléthorique de l'effectif des élèves dans les salles de classes (57 élèves pour 1 classe à Céchi, 51 élèves pour 1 classe à Loviguié au primaire et 65 élèves pour 1 classe au niveau du département). Dans le domaine de la santé, on relève 1 ESPC pour 18625 habitants dans la sous-préfecture d'Aboudé-Mandeké, 1 ESPC pour 13163 habitants à Agboville et ESPC pour 12419 habitants à Rubino. Ces problèmes résultent de la minorité des projets réalisés par rapport à l'effectif global des projets programmés. Cette situation est liée à la faiblesse de l'ensemble des dotations budgétaires de ces collectivités territoriales, au contexte économique et sociodémographique du département d'Agboville.

\section{References:}

1. BOSSON Eby 2013. Contrastes de développement dans l'ancienne boucle du cacao en Côte d'Ivoire : cas des départements d'Adzopé et de Bongouanou ; Abidjan, UFHB, Cocody, Thèse de Doctorat de $3^{\text {ème }}$ cycle, $439 p$

2. CHAMBAS Gérard, BRUN Jean-François et GRAZIOSI Grégoire, 2007. La mobilisation de ressources propres locales en Afrique. Forum « Décentralisation et gouvernance locale » / Nations Unies, Vienne, 27p.

3. Conseil café-cacao, 2016. Evolution des superficies et des productions de café-cacao dans le département d'Agboville ; $2 \mathrm{p}$

4. DCGTx, 1994. Plan d'Urbanisme Directeur d'Agboville ; Rapport d'enquête ; Abidjan, 72p.

5. DUBRESSON Alain, 1981. Régionalisation de l'industrie et croissance urbaine : le « mammouth » Cotivo à Agboville (Côte d’Ivoire) ; Cahier ORSTOM, sér. Sci. Hum., vol XVIII, n¹, pp 149164

6. HOUNSOUNON Damas 2016. Décentralisation et qualité de l'offre de services socio-publics en Afrique subsaharienne ; Afrobarometer, Working Paper $\mathrm{N}^{\circ} 165,30 \mathrm{p}$

7. INS, 1998. Recensement Général de la Population et de l'Habitation (RGPH, 1998) ; Vol IV : Tome 1 : état et structures de la population ; $118 \mathrm{p}$.

8. INS, 2014. Recensement Général de la Population et de l'Habitat, résultats globaux; secrétariat technique permanent du comité technique du RGPH ; $25 \mathrm{p}$

9. KONAN Pascal, 2011. Développement urbain en Côte d'Ivoire : cas de la ville de Dimbokro ; Thèse de Doctorat Unique en Géographie, Université de Cocody, 336p. 
10. KOUADIO Akou Aka, 2010. L'accessibilité des populations rurales aux soins de santé dans le département d'Abengourou (Côted'Ivoire) », Les Cahiers d'Outre-Mer, p. 439-459.

11. LOBA Valery 2008. Dynamique du développement des villes côtières dans la région des lagunes : cas de Bingerville, Dabou et Grand-Lahou ; Thèse de Doctorat Unique en Géographie, Université de Cocody, $388 \mathrm{p}$.

12. LOBA Valéry, 2009. La problématique de la couverture en structures sanitaire dans la région des lagunes ; Revue de Géographie Tropicale et d'Environnement, ${ }^{\circ} 1$, pp 21-32

13. MEMEL Frédéric, 2012. Ressources communales et aménagement urbain en Côte d'Ivoire : cas de la ville de Dabou; Thèse de Doctorat en Géographie, Université de Cocody, 352p.

14. MINISTERE de l'Economie, des Finances et du Plan, 1981. La Côte d'Ivoire en chiffres 1979-1980, Abidjan, SAE, 319 p.

15. MINISTERE du Plan et du Développement, 2015. Etude monographiques et économiques des Districts de Côte d'Ivoire (PEMED-CI) ; 305p

16. NGAMASSU David, 2005. Problématique des grands groupes et didactique du français au Cameroun ; Corela, Vol. 3, $\mathrm{n}^{\circ} 1 ; 23 \mathrm{p}$.

17. NKENGFACK Hilaire, DOMGUIA Edmond et KAMAJOU François, 2017. Analyse des déterminants de l'offre de l'eau potable au Cameroun ; <hal-01510111>, 21p.

18. OVONO Alexis, 2012. L'expérience de la Côte d'Ivoire en matière d'emprunt et/ou de subvention aux collectivités locales; Colloque l'autonomie financière des collectivités locales en Afrique noire francophone. Le cas du Cameroun, de la Côte d'Ivoire, du Gabon et du Sénégal ; 24p

19. PARE-KABORE Afsata et GAMBRE-IDANY Aïssa, 2014. Effectif par classe, performance scolaire des élèves et stratégies de gestion des grands groupes au Burkina Faso; Revue internationale de communication et de socialisation ; Vol 1, $\mathrm{n}^{\circ}$ 2, pp 125-171.

20. MINISTERE de l'Agriculture, 2007. Recensement National de l'Agriculture 2001. Région de l'Agneby ; Abidjan, Caistab, 73p.

21. RUF François et AGKPO Jean Luc. 2008. Etude sur le revenu et les investissements des producteurs de café et de cacao en Côte d'Ivoire : rapport final. Mai $2008 ; 115 \mathrm{p}$.

22. TOUZI Rachid, 2000. La décentralisation fiscale en Afrique : Succès, Problèmes et Contraintes ; Windhoek, 6p.

23. YATTA François Paul, 2009. La décentralisation fiscale en Afrique - Enjeux et perspectives; Karthala, collection économie et développement, 324p. 
24. YATTA François Paul, 2015. Le développement économique local en Afrique, mise en œuvre, contraintes et perspectives ; Platforma ; 25p.

25. ZAID-CHERTOUK Ahmed, 2007. Le développement local à travers une analyse critique des finances communales de la wilaya de TiziOuzou ; Revue Campus, n5, $21 \mathrm{p}$. 